\title{
Source Data Verification
}

Source Data Verification (SDV) is a required process to set up the reliability and evaluation of the collected data in clinical studies.

\section{Regulatory Reference}

! ICH GCP Guideline, Chapter 5.18.4

\section{Responsibilities}

\section{Purpose}

SDV is a process to verify CRF-entries against data in the source documents (original documents, data and records). Performed by the Monitor at each site-monitoring visit, all deviations and discrepancies in the data have to be documented and reported to the sponsor-investigator and related staff.

\section{Source Data Verification and Related Activities}

The following issues describe the sponsor-investigator's responsibilities related to SDV. This procedure is completed upon the initial review of the CRF and attached to the Site Monitoring Report:

Initial verification of critical data (completed during first Site Monitoring Visit):

- Informed consent obtained prior to inclusion in study

- Visit date(s) as available

- Eligibility criteria

- Medical history

- Medication history

Ongoing verification of critical data (completed during subsequent site monitoring visits):

- Visit date

- AEs

- SAEs

- Concomitant medications

- Study drug dispensing

- Answered queries pertaining to key efficacy parameters, changes re-confirmed with the source documents

- Primary efficacy data

Ongoing verification of non-critical data, if needed (to be completed during subsequent site monitoring visits).

\section{Forms and Templates}

Source Data Verification Form 